\title{
Douleur et Francophonie : divergences et convergences
}

\section{Pain and the Francophone population: variations and similarities}

\author{
A. Berquin \\ (C) Springer-Verlag France 2012
}

Y a-t-il une spécificité francophone dans l'abord des douleurs ? Tenter de répondre à cette question, c'est d'abord faire face à une difficulté sémantique. S'il est déjà ardu de définir la douleur, que proposer comme définition de la Francophonie? En dehors de ses utilisations politicoéconomiques, que certains qualifient de néocolonialistes, ce concept recouvre des situations concrètes très diverses. En France, le français est la langue maternelle de la majorité de la population. Dans d'autres pays (Suisse, Belgique, Canada), le français est une langue majoritaire dans certaines régions uniquement. Enfin, le français est plutôt une langue administrative ou une seconde langue dans un troisième groupe de pays (Luxembourg, certains pays d'Afrique).

Face à ces diversités, on peut s'attendre à ce qu'il soit difficile, voire impossible, de dégager une spécificité francophone dans l'approche des douleurs. C'est le constat que fait le Dr Lossignol, qui rappelle que les distinctions selon l'ethnicité, le genre ou la langue sont plus des constructions sociales qu'une réalité scientifique et qu'il peut y avoir moins de différences entre deux personnes de groupes distincts qu'entre deux personnes du même groupe.

Pourtant, des collègues béninois décrivent des modulations de l'expression de la douleur selon les ethnies. Étonnamment, ces différences sont décrites dans la vie communautaire mais sont absentes dans la vie professionnelle. Les influences du contexte culturel sur l'expression de la douleur doivent donc être abordées avec précaution et sous le signe de la complexité.

S'il est un terme important pour décrire la douleur chronique, c'est en effet bien celui de la complexité. C'est la raison pour laquelle nos collègues suisses rappellent l'importance de l'interdisciplinarité dans l'abord de la douleur. Ils en décrivent trois déclinaisons, notamment au sein de la Société suisse pour l'étude de la douleur.

Les sociétés scientifiques sont un moteur important de l'amélioration de la prise en charge de la douleur. Le Cercle

\footnotetext{
A. Berquin $(\triangle)$

Cliniques universitaires Saint-Luc, consultation de la douleur chronique, avenue Hippocrate 10/1650, B-1200 Bruxelles, Belgique e-mail : anne.berquin@uclouvain.be
}

luxembourgeois d'algologie a été fondé récemment. Parmi ses objectifs, relevons la négociation avec les autorités de tutelle dans le but d'obtenir une structuration et un financement adéquat de la prise en charge de la douleur.

L'importance de l'action politique pour promouvoir l'amélioration des soins en matière de douleur explique l'implication d'autres sociétés scientifiques nationales dans des négociations avec leurs pouvoirs publics respectifs, avec divers résultats concrets. En Belgique, des projets pilotes ont été peu à peu mis en place depuis 2005 ; ils ont récemment fait l'objet d'une évaluation, et les instances compétentes planchent actuellement sur un projet d'organisation structurelle des soins de la douleur. Au Québec, une démarche rigoureuse impliquant tous les acteurs et décideurs a permis de définir et valider une vision commune, puis de planifier le développement progressif des structures nécessaires et enfin de réfléchir à un dispositif d'évaluation et d'amélioration de la qualité. En France enfin, nul n'ignore l'action volontariste menée par les pouvoirs publics, en partenariat étroit avec la Société française d'étude et de traitement de la douleur (SFETD), depuis plus de 15 ans, et les «plans douleur » qui ont permis une amélioration progressive de l'offre de soins et de l'enseignement.

Ces descriptions - non exhaustives - des initiatives concernant l'organisation des soins de santé dans le domaine de la douleur, essentiellement chronique, dans plusieurs pays où on parle français montrent moins de divergences que de convergences.

Certes, les contraintes socio-économiques et géographiques peuvent être très différentes et influencer l'accessibilité des soins (citons comme seul exemple la densité de la population : 360 habitants $/ \mathrm{km}^{2}$ en Belgique, six habitants $/ \mathrm{km}^{2}$ au Québec). Mais les modèles d'organisation des soins dans le domaine de la douleur sont très comparables et - gageonsle - nullement différents de ceux envisagés dans les pays anglo-saxons. Les mêmes mots clés sont retrouvés : modèle biopsychosocial et multidisciplinarité, niveaux de soins, continuum de services, importance de l'enseignement et de la recherche, équilibre entre centralisation et décentralisation.

Surtout, relevons partout une implication forte de tous les professionnels concernés pour œuvrer à l'amélioration de l'accompagnement des personnes souffrant de douleurs. 